Journal of Mathematics and Statistics 1 (3): 203-211, 2005

ISSN 1549-3644

(c) 2005 Science Publications

\title{
Hemisphere Sizing from Linear Intercept Measurement
}

\author{
Wilfried Gille \\ Martin-Luther-University Halle-Wittenberg \\ Department of Physics, SAS-Laboratory, Hoher Weg 8, D-06120 Halle, Germany
}

\begin{abstract}
The stereological problem of unfolding the hemisphere radius distribution from the chord length distribution is analyzed. Let a stationary isotropic process of hemispheres be given. The hemispheres have random diameters and are isotropically uniformly randomly orientated in space. A straight line probe yields a process of intercepts. The inverse problem of re-obtaining the size distribution of the hemispheres in terms of an experimental intercept length distribution is solved. The chord length distribution of a single hemisphere, known analytically, is approximated by piecewise polynomials in two intervals. The solution of the inverse problem is traced back to a simple recurrence equation. Numerical checks with exact and simulated data are performed to demonstrate the applicability. Data of "chord length sampling", resulting from image analysis procedures, from scattering methods or from other appropriate physical apparatuses, are applicable.
\end{abstract}

Key words: Stereology, inverse problem, IUR chords, size distribution, recurrence equation

\section{INTRODUCTION}

One of the fundamental problems in stereology is the derivation of the size distribution of geometric objects (particles) from partial information which is contained in the length distribution of linear intersections of the particles (Fig. 1). Textbooks by Weibel $^{[1]}$, Stoyan, Kendall and Mecke ${ }^{[2]}$, Serra ${ }^{[3]}$, Ohser and Mucklich ${ }^{[4]}$ handle this problem for specific shapes. Information about the particle size via chord length measurement can be obtained by physical apparatuses.

The standard problem of tracing back the size distribution of hemispheres to linear intercept measurement occurs in different fields, involving specificities. The universal nomenclature used in this representation is explained in Appendix A.

Chord length distributions (CLDs) were investigated by Enns and Ehlers ${ }^{[5]}$. Applications of particle sizing in materials science were discussed by Hermann $^{[6]}$. Scattering methods, especially small-angle scattering (SAS), Appendix B, were applied for particle sizing on the nanometric length scale ${ }^{[7-9]}$.

Let $L$ be the largest diameter of the largest hemisphere. For the analysis of the hemisphere sizes, isotropic uniform random chord lengths exclusively inside the hemispheres will be analyzed, $0 \leq r \leq L$. No outside chord lengths or any other experimental information about the spatial arrangement of the hemispheres are included and no interaction between the hemispheres is considered.

For the following, the normalized, isotropized geometric covariogram of the hemispheres will serve as a universal working function (WF) $\gamma(r), \gamma(0)=1$, $0 \leq r<L, \gamma(r) \equiv 0$ if $L<r$,

$\gamma(r)=\frac{K(r)}{K(0)}$

Here, the function $K(r)=\overline{K(|\vec{r}|)}$ is the isotropized geometric covariogram of an ensemble of hemispheres. Some steps of explanation concerning the WF and the background of Eq. (1) are helpful.

The consideration starts with the geometric covariogram $K_{1}(\vec{r})$ for a single particle in a fixed direction ${ }^{[3]}$. The geometric covariogram is useful to characterize a single hemisphere $H_{R}$ of radius $R$, as well as an ensemble of hemispheres possessing a certain size distribution.

For a single hemisphere the geometric covariogram $^{[3]}$ is the overlapping integral $K_{1}(\vec{r})=V\left(H_{R} \cap\left(H_{R}+\vec{r}\right)\right)=\int 1_{H_{R}}(\vec{x}) \cdot 1_{H_{R}}(\vec{x}+\vec{r}) d \vec{x}, \vec{r} \in \mathfrak{R}^{3}$, where $1_{H_{R}}$ is the indicator function of $H_{R}$ and $V$ the overlapping volume. The function $K_{1}(\vec{r})$ describes the overlapping in a fixed direction.

Further, the isotropized covariogram of $H_{R}$, $\bar{K}_{1}(r)$, follows from spatial averaging over the spherical unitshell of surface area $4 \pi$ in $\mathfrak{R}^{3}$,

$\overline{K_{1}(|\vec{r}|)}=\bar{K}_{1}(r)=1 /(4 \pi) \cdot \int K_{1}(r \cdot \vec{u}) d \vec{u}=$

$1 /(4 \pi) \cdot \int_{-\pi / 2}^{\pi / 2} \int_{0}^{2 \pi} K_{1}(r(\vec{u}(\varphi, \vartheta))) \cdot \cos (\vartheta) d \varphi d \vartheta, 0 \leq r$.

Corresponding Author: Wilfried Gille, Martin-Luther-University Halle-Wittenberg, Department of Physics, SASLaboratory, Hoher Weg 8, D-06120 Halle, Germany 
Here, $d \vec{u}$ is the element of the surface measure, given by use of spherical coordinates $\varphi$ and $\vartheta$ via $\vec{u}(\varphi, \vartheta)=(\cos (\varphi) \cdot \cos (\vartheta), \sin (\varphi) \cdot \cos (\vartheta), \sin (\vartheta)) \cdot$

The function $\bar{K}_{1}(r)$ possesses the property $\bar{K}_{1}(0)=K_{1}(\overrightarrow{0})=V\left(H_{R}\right)$. Considering a single hemisphere $H_{R}$, it is useful to define a dimensionless working function $\gamma_{1}(r)=\bar{K}_{1}(r) / \bar{K}_{1}(0), 0 \leq r$. Evidently, $\gamma_{1}(r)$ is a strictly monotonously decreasing function on the interval $0 \leq r \leq 2 \cdot R$, possessing the property $4 \pi \cdot \int_{0}^{2 \cdot R} r^{2} \cdot \gamma_{1}(r) d r=V\left(H_{R}\right)$. The function $\gamma_{1}(r)$ starts in the origin with $\gamma_{1}(0)=1$.

In the case of an ensemble of $N$ differently sized hemispheres (volumes $V_{1,2,3, \ldots}$ ), there exist $N$ functions $\gamma_{1_{i}}(r), i=1,2,3 \ldots N$. The function $\gamma_{1_{i}}(r)$ describes the $i^{\text {th }}$ hemisphere with volume $V_{i}$. The averaged working function $\gamma(r)$ is defined by the ratio $\gamma(r)=\sum_{i=1}^{N}\left[V_{i} \cdot \gamma_{1_{i}}(r)\right] / \sum_{i=1}^{N}\left[V_{i}\right]$. Being a sum of strictly monotonously decreasing functions, $\gamma(r)$ is again strictly monotonously decreasing. Further, the WF $\gamma(r)$ is dimensionless, real valued and possesses the property $0 \leq \gamma(r) \leq 1$.

Operating with $\gamma(r)$, an isotropic and homogeneous arrangement of homogeneous hemispheres with random radius $R$, distribution function $F(R)$ and continuous distribution density function $f(R)=F^{\prime}(R)$, is analyzed (Fig. 1).

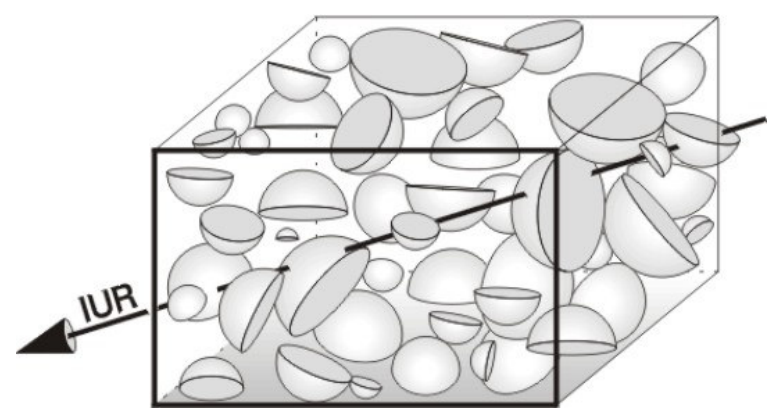

Fig. 1: Particle sizing of hemispheres of random size via analysis of the length distribution law of linear intercepts: Assuming an isotropic geometric arrangement, any testline can be applied for measurement of IUR chord lengths inside the particles

Let $R_{\max }$ be the largest radius. The volume fraction of the hemispheres inside the sample volume is an insignificant parameter, $\gamma(r) \equiv 0$, if $L=2 R_{\max }<r$. The functions $K(r)$ and $\gamma(r)$ are assumed to be exclusively influenced by the sizes of the hemispheres. Clearly, this is trivial in cases, if image material is available and exclusively chord lengths inside the hemispheres can be selected and measured. However, in the case of scattering experiments, the assumption of a so-called quasi-diluted particle arrangement must be additionally fulfilled, Appendix B (Fig. B1).

For compact convex particles, the chord length distribution density $A(l)=A(l, R)$ is connected with $\gamma(r)$

$\gamma(r)=\frac{\int_{r}^{L}(l-r) \cdot A(l) d l}{\int_{0}^{L} l \cdot A(l) d l}, A(l)=\gamma^{\prime \prime}(l) \cdot \int_{0}^{L} l \cdot A(l) d l=\gamma^{\prime \prime}(l) \cdot \bar{l}$

look for example back at the parallelepiped case ${ }^{[10]}$. Thus, $A(l)$ defines $\gamma(r)$. In the other direction, $\gamma(r)$ defines $A(l)$ via the second derivative. From geometric principles, $A(l)$ functions were determined for many basic geometric shapes ${ }^{[5,11-13]}$.

Particularly, the analytical expression for the CLD of the hemisphere with a fixed radius $R, A_{H}(l, R)$, is known, Gille ${ }^{[10,14]}$ (Fig. 2). The motivation of these calculations are experimental results, showing the existence of hemispherical micro-particles, Appendix C. Moreover, there is a theoretical interest to add a new result to the sequence of known cases with elementary particle shapes.

There are cases in which two- or three-dimensional images are available. The theory reported here, operating with the WF $\gamma(r)$, can even be applied without an image.

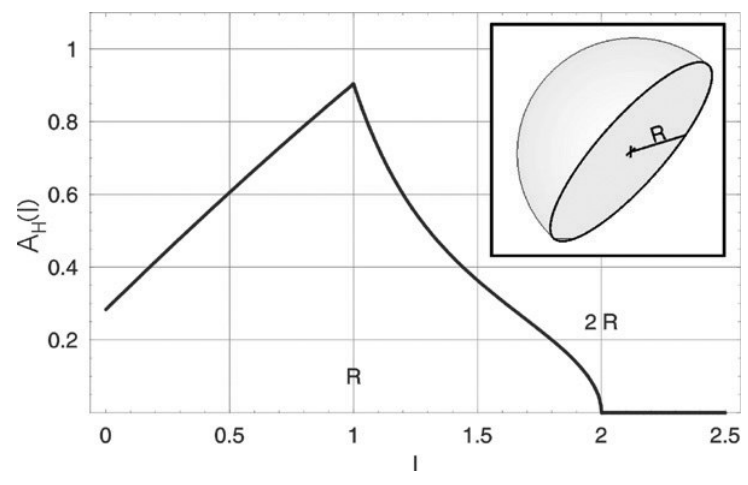

Fig. 2: Isotropized chord length distribution density $A_{H}(l)=\bar{l} \cdot \gamma_{H}{ }^{\prime \prime}(l)$ of a single hemisphere of radius $R=1, L=2$

This project starts with the analysis of the relation between $f(R), \mathrm{WF}$ and CLD, operating with the analytical expression $\gamma_{H}(r, R)$ for the single hemisphere of radius $R$ (Fig. 2). Section 2 considers the general formulas for the simulation of the WF and the CLD depending on $f(R)$. Section 3 analyses the backstep, $f(R)$ in terms of $\gamma(r)$. However, as it seems to be impossible to find an exact solution, sections 3.1-3.3 
consider a suitable approximation of $\gamma_{H}$. A numerical test of this approximation is included.

Finally, a recurrence equation follows, connecting $f(R)$ and $A(l)$, sections 3.4, 3.5. After some checks an application for a simulated case follows. Selected steps of the project are explained in greater detail in the Appendices.

\section{Simulation of $\gamma(r)$ from $f(R)$}

2.1 The hemisphere correlation function $\gamma_{H}(r, R)$ : For a single hemisphere of radius $R$ (for more details Gille $^{[14]}$ and Appendix D),

$$
\begin{aligned}
& \gamma_{H}(r, R)=\left|1-\frac{9 r}{8 R}+\frac{r^{3}}{(2 R)^{3}}+\frac{3 \cdot\left(r^{2}-R^{2}\right) \cdot \arcsin [r /(2 R)]}{4 \pi \cdot r R}\right|_{0}^{R} \\
& +\left|\frac{3 \cdot\left(r^{2}+2 R^{2}\right) \sqrt{4 R^{2}-r^{2}}}{32 \pi \cdot R^{3}}\right|_{0}^{2 R}+\left|\frac{3 \cdot\left(R^{2}-r^{2}\right) \cdot \arccos [r /(2 R)]}{4 \pi \cdot r R}\right|_{R}^{2 R} \cdot(3)
\end{aligned}
$$

The abbreviation for the interval restrictions in Eq. (3) means

$$
|\operatorname{term}(x)|_{x_{1}}^{x_{2}}=\left\{\begin{array}{cll}
\operatorname{term}(x) & , \text { if } & x_{1} \leq x \leq x_{2} \\
0 & , & \text { else }
\end{array}\right.
$$

Thus, $\gamma_{H}$ is split into three functions: $\gamma_{H 1}(r, R)$ if $0 \leq r \leq R ; \gamma_{H_{2}}(r, R)$, if $R \leq r \leq 2 R ; \gamma_{H 3}(r, R)=0$ if $2 R<r$.

2.2 Averaging over different radii: The volume $R^{3} \cdot \gamma_{H}(r, R)$ is proportional to the geometric covariogram, Serra ${ }^{[3]}$. Then, the averaged WF $\gamma(r)$ is defined in terms of $f(R)$ and $\gamma_{H}(r, R)$

$\gamma(r)=\frac{\int_{\frac{r}{2}}^{R_{\max }} R^{3} \cdot \gamma_{H}(r, R) \cdot f(R) d R}{\int_{0}^{R_{\max }} R^{3} \cdot f(r) d R}$,

$0 \leq r \leq 2 \cdot R, \quad 0 \leq R \leq R_{\max }$.

The integral in the denominator of Eq. (5) is the third moment M3 of $f(R)$. The ratio $R^{3} \cdot \gamma_{H}(r, R) / M 3$ is the weighing function for averaging. Eq. (5) satisfies $\gamma(0)=1$. The lower integration limit depends on $r$. Here, $r / 2 \leq R$ is necessary to fulfil $0 \leq r \leq 2 \cdot R$, including the condition $r \leq 2 R_{\max }$, Eqs. $(3,4)$. In more detail, for any assumed $f(R)$, the averaged WF is a sum of two parametric integrals

$$
\begin{aligned}
& M 3 \cdot \gamma(r)= \\
& \int_{r}^{R_{\max }} R^{3} \cdot \gamma_{H 1}(r, R) f(R) d R+\int_{\frac{r}{2}}^{r} R^{3} \cdot \gamma_{H 2}(r, R) f(R) d R .
\end{aligned}
$$

The mean hemisphere volume $\bar{V}$ is $\bar{V}=2 \pi / 3 \cdot M 3$. Eq. (6) satisfies $\gamma\left(R_{\max }\right)=0$. The connection between Eq. (6) and $A(l)$ is Eq. (2). Furthermore, the WF can be traced back to the isotropic scattering intensity, Appendix B.

\subsection{An assumed density function $f(R)$ :}

Let $f(R)$ be the superposition of two Maxwell distribution densities with the first moments $m_{x 1}=15$ and $m_{x 2}=50$ (two local maxima occur in Fig. 3).

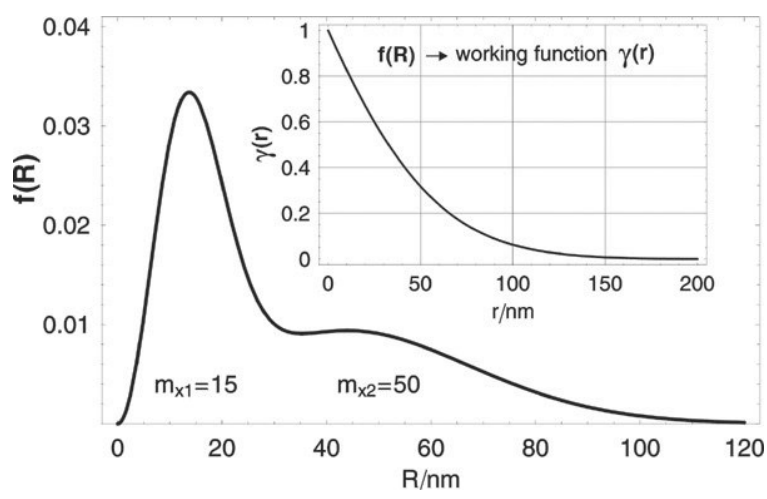

Fig. 3: Superposition of two Maxwell distribution densities: $f(R)$ extends from 2 to $R_{\max }=120$. The WF in the insert is simulated numerically, $0<r<200$, Eq. (6)

$f(R)=\frac{1}{2} \cdot\left(a_{1} R^{2} \cdot e^{-\alpha_{1}^{2} \cdot R^{2}}+a_{2} R^{2} \cdot e^{-\alpha_{2}^{2} \cdot R^{2}}\right)$,

$\alpha_{1,2}=\frac{2}{m_{x 1,2} \cdot \sqrt{\pi}}, a_{1,2}=\frac{32}{\pi^{2} \cdot m_{x 1,2}^{3}}$

Consequently, $M 3 \approx 10^{5}$ and $\bar{V}=2.1 \cdot 10^{5}$. The insert of Fig. 3 involves the resulting WF $\gamma(r)$, Eqs. $(6,3)$. The WF curve, given in the limited interval, $0 \leq r \leq 200$, results. An existing truncation error $(\gamma(r) \equiv 0$, if $200<r)$ can be neglected. The WF defines the CLD via Eq. (2). On the other hand, the SAS curve of the polydisperse hemisphere system (Fig. B2) involves the same information about $f(R)$, but it is based on another experimental (sampling) technique.

This way, depending on a special experimental sampling technique used, the information about the unknown function $f(R)$ is contained in the WF as well as in the CLD and as well as in the SAS curve.

Now, the inverse problem consists in the determination of $F(r), f(R)$ based on $A(l)$. Depending on the respective practical problem to be solved, $A(l)$ can be measured directly or obtained from the WF. Furthermore, the WF can be traced back to the scattering intensity.

\section{Solution of the inverse problem}

3.1 Analysis of Eq. (6): The functions $f(R), \gamma(r)$ and $\gamma_{H}(r, R)$ are connected via Eq. (5), or in greater detail, 
by Eq. (6). Taking into account the current opinion of the specialists in the field of integral equations, for example Camko et al. ${ }^{[15]}$, Wiener ${ }^{[16]}$, including the actual developments in the field of computer algebra, Mathematica $^{[17]}$, it seems difficult to find out an exact analytical solution. Fedorova \& Schmidt ${ }^{[11]}$ reached analytic solutions for particle sizing problems for a sequence of particle shapes. The hemisphere case is not included there. There are no simplifying integral transformations for the actual kernel type. Evidently, the only way is to apply numerical procedures, developed for solving a wide group of inverse problems.

Another way for solving the inverse problem is to apply a method recently developed by Ohser, also Ohser \& Mucklich $^{[4]}$. This procedure should work for all cases, when the kernel function is known explicitly with high precision (which is fulfilled by Eq. (3)).

However, in the present paper the goal of developing a simple practicable solution was reached by puzzling over a suited approximation of the function $\gamma_{H}(r, R)$. In the beginning, it seemed to be advisable to apply series expansions, Gille ${ }^{[10]}$, or their combination at well chosen $r$-positions. A two-dimensional series expansion of $\gamma_{H}(r, R)$ was also inserted into Eq. (6).

A long series of trials has shown that the best suited approximation (with respect to the desired solution of Eq. (6)) is a piecewise linear approximation on two $r$-intervals. This is based on the fact that the CLD (Fig. 2) can be approximated by a straight line in the first $r$ interval, $0 \leq r \leq R$. After two differentiation steps (operating with Eqs. (3-6)), the second derivative $\gamma^{\prime \prime}(r)$ of the WF is

$$
\begin{aligned}
& M 3 \cdot \gamma^{\prime \prime}(r)= \\
& \int_{r}^{R_{\max }} R^{3} \cdot \gamma_{H 1}^{\prime \prime}(r, R) f(R) d R+\int_{\frac{r}{2}}^{r} R^{3} \cdot \gamma_{H 2}^{\prime \prime}(r, R) f(R) d R
\end{aligned}
$$

For both differentiation steps, the limiting relations $\gamma_{H}\left(2 \cdot R_{\max }, R\right)=0, \quad \gamma_{H}^{\prime}\left(2 R_{\max }+, R\right)=0 \quad$ and $\gamma_{H}^{\prime \prime}\left(2 R_{\max }+, R\right)=0$ are essential. Consequently, the unknown function $f(r)$ is defined in terms of the transformation Eq. (6) and as well in terms of Eq. (8). Thus, Eqs. $(6,8)$ are equivalent.

Furthermore, the left hand side functions $f(r)$ and $\gamma^{\prime \prime}(r)$ can be traced back to experimental values. Data from linear intercept measurements define the left hand side of Eq. (8). According to Eq. (2), $A(r)$ is proportional to $\gamma^{\prime \prime}(r)$ on the whole interval $0 \leq r \leq 2 \cdot R_{\max }$. No matter which of the functions, $\gamma(r)$ or $\gamma^{\prime \prime}(r)$ or $A(r)$ is given experimentally, Eq. (8) is the equation to be inverted in order to determine $f(R)$.

3.2 Approximation of $\gamma_{H}(r, R)$ by a function: $\gamma_{A}(r, R)$ : Let $\gamma_{A}(r, R)$ be the approximation in question of the exact function $\gamma_{H}(r, R)$. Considering the intrinsic properties of the CLD (Fig. 2) in greater detail, two polynomials of third degree on the $r$-intervals, $0 \leq r \leq R$ and $R \leq r \leq 2 \cdot R$ fulfil $\gamma_{H}(r, R) \approx \gamma_{A}(r, R)$,

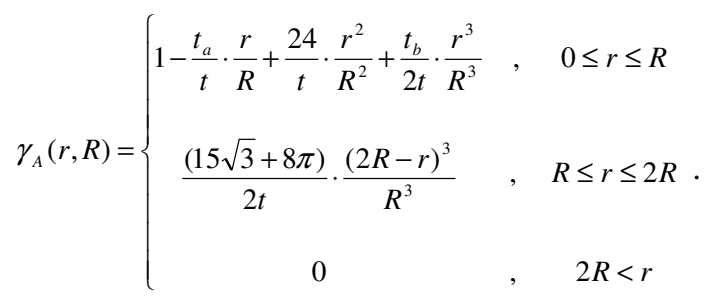

Eq. (9) uses the abbreviation $t=8+45 \sqrt{3}+24 \pi$, $t_{a}=3(8+15 \sqrt{3}+8 \pi), t_{b}=(15 \sqrt{3}+8 \pi-16)$. The degree of the $r$ polynomial applied is a compromise. The approximation fulfils $\gamma_{A}(0, R)=1$. Differentiation of the terms in Eq. (9) with respect to $r$ yields the terms of $\gamma_{A}{ }^{\prime \prime}(r, R)$, Appendix E. Altogether, Eq. (8) is simplified.

Numerical methods for solving integral equations are not applied. By use of Eq. (9), Eqs. (6,8) simplify by $r$-differentiation. After this intermediate step, $f(R)$ is defined implicitly.

3.3. The solution for $f(r)$ : Operating with Eq. (9), a form involving the distribution function, containing terms $F(r)$ and $F(r / 2)$ is obtained after three differentiation steps

$$
\begin{aligned}
& \begin{array}{l}
M 3 \cdot \gamma^{(3)}(r)= \\
\left\{\left(-3(15 \sqrt{3}+8 \pi) \cdot\left[F(r)-F\left(\frac{r}{2}\right)\right]+\right.\right. \\
3(-16+15 \sqrt{3}+8 \pi) \cdot[1-F(r)]\} /(8+45 \sqrt{3}+24 \pi)
\end{array} .
\end{aligned}
$$

Inserting $r=0$ and $F(0)$ into Eq.(10) yields,

$M 3 \cdot \gamma^{(3)}(0)=\frac{3(-16+15 \sqrt{3}+8 \pi)}{8+45 \sqrt{3}+24 \pi}=0.6529$

Eq. (11) defines M3. Furthermore, the differentiation step $f(R)=F^{\prime}(R)$ transforms Eq. (10) into a recurrence equation

$$
\begin{aligned}
& \frac{M 3}{3} \cdot \gamma^{(4)}(r)=c_{1} \cdot f\left(\frac{r}{2}\right)+c_{2} \cdot f(r)= \\
& \frac{(15 \sqrt{3}+8 \pi) \cdot f\left(\frac{r}{2}\right)-4(-8+15 \sqrt{3}+8 \pi) \cdot f(r)}{16+90 \sqrt{3}+48 \pi} .
\end{aligned}
$$

This result, Eq. (12), is remarkable. The function $f$ is traced back to the WF $\gamma(r)$. In fact, $\gamma^{(4)}$ is proportional to the second derivative $A^{\prime \prime}(r)$ of the 
experimental chord length distribution density $A(r)$, Eq. (2).

3.4 A procedure for determining $f(R)$ : Because of experimental errors, a lower limit of information, $r=r_{0}$, exists. This has to be taken into account for establishing a stable procedure, based on Eq. (12). For example in image analysis $r_{0}$ is given by the resolution limit of the image. On computer screens $r_{0}$ is connected with the number of pixels used. For micro-particles in the field of SAS, $r_{0} \approx 2 \mathrm{~nm}$. On the other hand, there is an upper experimental limit $r_{\max }$. Consequently, limiting conditions $f(R)=0$, if $R<r_{0}$ and $f(R)=0$, if $r_{\max }<R$ exist. Additionally, there are noise terms, inherent in a data set, $\left\{l_{i}, A_{i}\left(l_{i}\right)\right\}, i=1, \ldots N$. In the simulated cases these parts have been obtained from random number generators.

The procedure $f\left[r_{-}, r 0_{-}\right]$involves these restrictions. The first step is the determination of M3 from the WF, followed by a recursive solution of the recurrence equation Eq. (12).

The algorithm is:

$t=8+45 \sqrt{3}+24 \pi ; \quad t 1=t-8 ; \quad t 2=12(15 \sqrt{3}+8 \pi-8) ;$

$t 3=3(15 \sqrt{3}+8 \pi-16)$;

$M 3=\frac{t 3}{t \cdot \gamma^{(3)}[r 0]}$;

(*COMMENT: recursive definition of $f[r, r 0]$, Eq. $\left.(12)^{*}\right)$

$f\left[r_{-}, r 0_{-}\right]:=$Which $[r<=r 0,0$,

$r 0<r<2 \cdot r 0, \frac{-2 \cdot M 3 \cdot t}{t 2} \cdot \gamma^{(4)}[r]$,

$\left.2 \cdot r 0<r<\infty,\left(t 1 \cdot f[r / 2, r 0]-2 \cdot M 3 \cdot t \cdot \gamma^{(4)}[r]\right) / t 2\right] ;$

(*COMMENT: The function Plot[] includes the recursion *)

Plot $\left[f[r, r 0],\left\{r, 0, r_{\max }\right\}\right]$;

The plot starts at $r=0$. If $r \leq r_{0}$, then 0 results until $r=r_{0}$ is reached. Then, for all the following actual $r$-values the term $t 1 \cdot f[r / 2, r 0]$ successively changes, according to Eq. (12), depending on $r_{0}$.

3.5 Application and stability: The given algorithm is applied to the simulated data set, section 2.3 and Fig. 3. The second derivative of the WF $\gamma^{\prime \prime}(r)$ is represented by a table $T=\left\{l_{i}, A_{i}\left(l_{i}\right)\right\}, i=1(1) 200$, whose numbers possess two digits. This represents a not normalized experimental CLD. Based on $T$, the function $f(R)$ should reappear. In fact, the dashed line (Fig. 4) is obtained, based on Eqs. $(11,12)$ which are involved in the procedure. The full line in Fig. 4 is given by Eq. (7).
Figure 4 demonstrates the stability of the method for the bimodal distribution type assumed. Furthermore, the procedure is checked for several conditions and for other size distribution models; Rayleigh-distribution, Normal-distribution, Log-Normal-distribution. The most sensitive point is the parameter $r_{0}$.

Exceptional situations can be constructed, in which the method fails (in the case of extremely narrow or not continuous distributions). Here, the approximation errors given by the difference between $\gamma_{H}$ and $\gamma_{A}$ (or $A_{H}$ and $A_{A}$ ) influence the stability.

In most of the tested cases the assumed $f(R)$ reappears, if $r_{0}$ is sufficiently small, compared with the abscissa value $R=R_{m}$ of the first maximum $f\left(R_{m}\right)$ of $f(R)$. Tests show that the relation $r_{0}<R_{m} / 5$ is sufficient. In most experimental cases, $r_{0}$ is known beforehand, however the parameter $R_{m}$ results (and can be checked) only after performing the data evaluation.

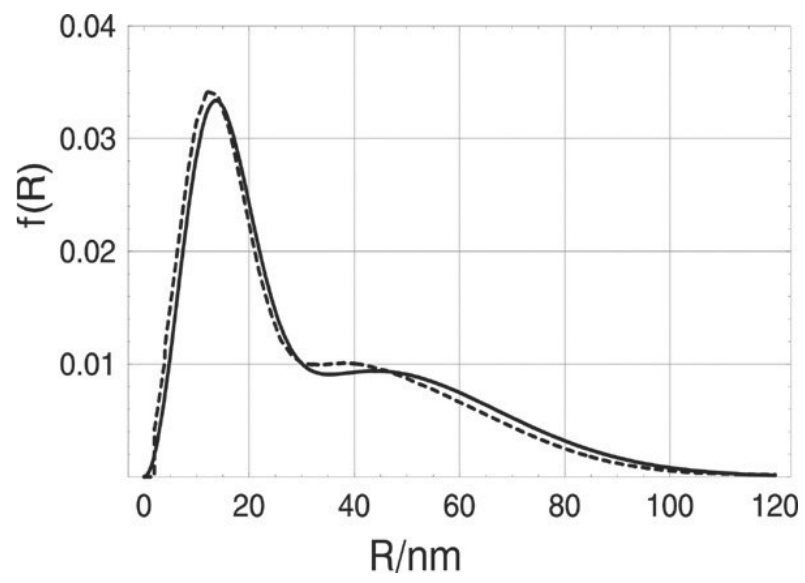

Fig. 4: Numerical test of the procedure in a special case, where $f(R)$ is a superposition of two Maxwell distribution densities (Fig. 3)

$f(R)$ (dashed line) is obtained, based on a table (200 simulated $\gamma^{\prime \prime}(r)$ values, each number with two digit precision). Here $r_{0}=2$ and $r_{\max }=200$ are inserted. Even in this case the procedure is applicable (logically, the smaller $r_{0}$ the better the dashed line agrees with Eq. (7)).

\section{CONCLUSION}

The problem of estimation of the radius distribution function and its density from the observation of chord lengths in IUR sections of a macroscopic isometric system of hemispheres with straight lines is solved. After writing the mean WF as an integral over the hemisphere size density, a procedure for determining $f(R)$ is established. This is 
based on the analytic expression for the single hemisphere chord length distribution and further, on the simplifying piecewise approximation of the WF by polynomials of third degree. Thus, a simple recurrence equation follows. It traces the unknown parameters back to a data table $T$, involving the frequency of chord segments $l_{i}$.

In most practical cases, smooth distributions without singularities exist. Under this condition, a sequence of tests shows: A stable solution is obtained based on at least $N=200$ pairs $\left\{l_{i}, A_{i}\left(l_{i}\right)\right\}$ on the interval $r 0<l_{i}<2 \cdot R_{\max }<r_{\max }$. The approximation error, inherent in the method, is unimportant in contrast to the errors resulting from the lower experimental limit $r=r_{0}$.

\section{ACKNOWLEDGEMENTS}

I am very grateful to Dr. Saxl for the useful hints concerning the representation and the choice of the symbols used. Furthermore, I thank the Wolfram Research-Mathematica team (Illinois) for support, Prof. Wiener (Halle) for basic remarks concerning the analytic (possibly explicit) solution of Eq. (5) and J. Morvaj for help and discussion. The micrograph (Fig. C1) demonstrates the existence of hemispherical particles in nature. This goes back to discussions with Prof. Lukac (Prague), whom I thank very much for submitting me some experimental results.

\section{Appendix A: Notation}

\section{$R$}

$F(R), f(R)$ distribution function and distribution density of the random variable $R$

M3

$V, V_{1}, V_{2}, V_{3}$

$\bar{V}$

$r, R_{\max } ; L$

$T$

$l, m$

$r_{0}, r_{\max }$

$R_{m}$

radius of a single hemisphere, random variable

the third moment of $f(R)$

volume; volumes of three hemispheres

mean hemisphere volume

distance, radius of the largest hemisphere;

$L$ is the greatest existing particle diameter;

table of data, $T=\left\{l_{i}, A_{i}\left(l_{i}\right)\right\}, \quad i=1(1) N$, $N>200$ which represents the (simulated) experimental chord length distribution, all numbers should involve two digits

within, $l$ and outside, $m$, of the hemispheres

lower and upper experimental limit for length measurement

abscissa of the first maximum of $f(R)$
$K(r) \quad$ isotropized geometric covariogram

$\gamma(r) \quad$ working function (WF)

$A(l), A(r)$ chord length distribution density (CLD), for IUR-chords, experimental linear intercept measurements yield $A(r)$ for certain segment lengths $r_{0}<r<r_{\max }$.

$\gamma_{H}(r, R) \quad$ isotropized and normalized set covariance of a single hemisphere with fixed radius $R$

$A_{H}(l, R) \quad$ CLD for a single isolated hemisphere with fixed radius $R$

$\gamma_{A}(r, R) \quad$ analytically approximated WF of a single hemisphere with fixed radius $\mathrm{R}$

$A_{A}(L, R)$ analytically approximated CLD of a hemisphere with fixed radius $R$

$t, t_{a}, t_{b}, c_{1}, c_{2}$ constants

$h \quad$ amount of the scattering vector

$I(h)$ isotropic scattering-intensity, normalization $I(0)=1$

\section{Appendix B: Scattering experiments}

Particle sizing by use of SAS experiments is a standard method, Guinier \& Fournet ${ }^{[18]}$. Here, the particle shape must be known a priori. No image material (micrograph) is necessary. Physical apparatuses record the (isotropic) scattering intensity curve $I(h)$ (scattering vector $h$ ). The WF can be traced back to $I(h)$, Torquato $^{[19]}$ (page 34, equations 2.30 and 2.31 in that textbook), by inversion of Eq. (13)

$I(h)=\frac{\int_{0}^{L} 4 \pi \cdot r^{2} \gamma(r) \cdot \sin (h \cdot r) /(h \cdot r) d r}{\int_{0}^{L} 4 \pi \cdot r^{2} \gamma(r) d r}$

However, in order to exclude the confusing influence of so-called interparticle interferences the conditions explained in Fig. B1 must be fulfilled (socalled quasi-diluted particle arrangement).

This figure shows an assumed configuration of hemispheres 1, 2, 3 with volumes $V_{1}, V_{2}, V_{3}$ and corresponding WFs $\gamma_{1}, \gamma_{2}, \gamma_{3}$. The diameter $2 R=l$ is smaller than the minimum of all possible distances $m$ between any points of the surface areas of the hemispheres, 1-2, 1-3, 2-3. In such a system, the WF in the interval $0 \leq r \leq 2 \cdot R_{\max }$ is the volume averaged mean, whatever the particle shape, $\gamma(r)=\left[V_{1} \cdot \gamma_{1}(r)+V_{2} \cdot \gamma_{2}(r)+V_{3} \cdot \gamma_{3}(r)\right] /\left(V_{1}+V_{2}+V_{3}\right)$. The normalization $\gamma(0)=1$ is fulfilled. 


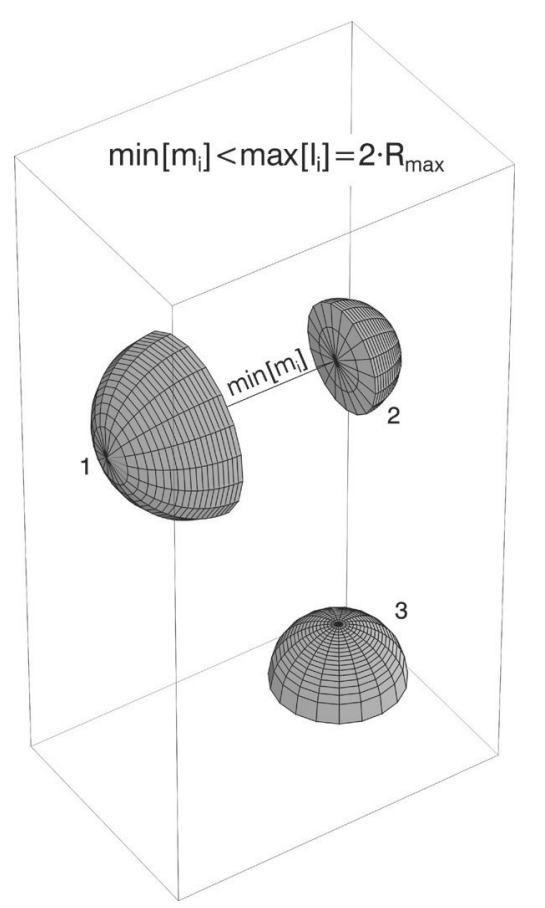

Fig. B1: Quasi-diluted conditions

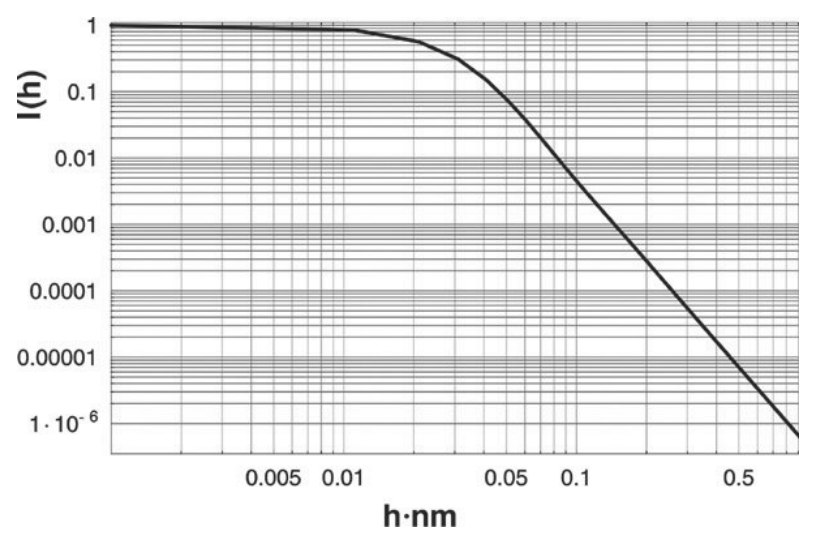

Fig. B2: Based on a simulation of the WF, $f(R)$ is given by Eq. (7), $I(h)$ is obtained via Eq. (13). In order to emphasize the high dynamics of the intensity, a logarithmic plot is used. Small hemispheres mainly influence $I(h)$ for large $h$. Big hemispheres are reflected with small $h$.

\section{Appendix C: Hemispherical particles in aluminum alloys}

There is an eminent physical background in materials research, Dutkievicz et al. ${ }^{[20]}$. This particle shape has already been observed in metal physics on a length scale of about $50 \mathrm{~nm}$ in several alloys. Lukac ${ }^{[21]}$ investigated the influence of the strain rate on the plastic instabilities in aluminum based alloys (Fig. C1).

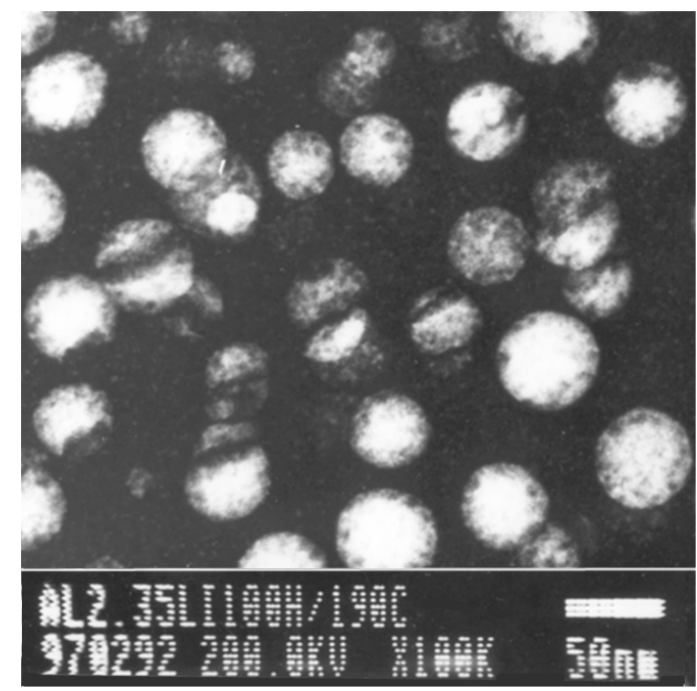

Fig. C1: Micrograph with hemispherical particles in $\operatorname{AlLi}(2.35 \%)$ material with a treatment: $T_{A}=190^{\circ} \mathrm{C}$ for $100 \mathrm{~h}$ (after homogenization)

Appendix D: Constellation for determining $A_{H}(l)$ of the hemisphere: Two types of chord lengths, basicchords (bc) and cap-chords (cc) (Fig. D1) must be distinguished. For reasons of symmetry, it is sufficient to consider one random angle $\alpha$, defining the interval limits of the IUR chords.

The area parts possible for $\mathrm{bc}$ and $\mathrm{cc}$ are related $2: 1$. The fundamental limits of $\alpha$ depend on the chord length $l$. Three cases have to be distinguished: case 1 : $\{0 \leq l<R, \quad 0 \leq \alpha<\pi / 2\}, \quad$ case $2: \quad\{R \leq l<\sqrt{2} \cdot R$, $0<\alpha<\arcsin (R / l)\}, \quad$ case $3: \quad\{\sqrt{2} \cdot R \leq l<2 \cdot R$, $0<\alpha \leq \arccos (l / 2 \cdot R)\}$. The distribution function $F(l)$, $F(l)=\operatorname{Pr}(\varsigma<l)$, can be expressed by ratios of projection portions perpendicular to the $\alpha$-direction. Finally, $A(l)=F^{\prime}(l)$ was performed, Gille $e^{[14]}$.

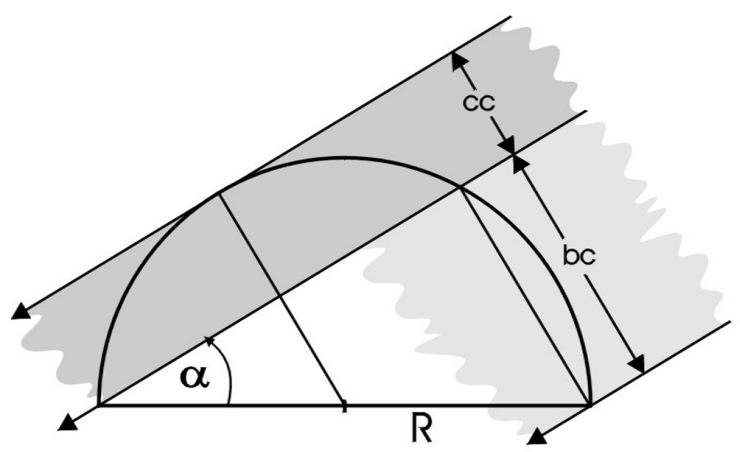

Fig. D1: Two basic cases: cc and bc. Consequently, the whole CLD is a superposition of two distribution densities $A_{c c}(l)$ and $A_{b c}(l)$, Fig. D2 
The analytic representation of $A_{b c}(l, R)$ is more complicated than that of $A_{c c}(l, R)$,

$$
A_{c c}(l, R)=\frac{2 l}{\pi R^{2}} \cdot \arctan \left(\sqrt{4 R^{2} / l^{2}-1}\right), \quad 0 \leq l \leq 2 R
$$

The first moment of $A_{c c}$ is $32 R /(9 \pi)$.

The superposition $A_{H}(l)=1 / 3 \cdot A_{c c}(l)+2 / 3 \cdot A_{b c}(l)$

(Fig. 2) takes into account the geometry of IUR-chords on a straight test line (Fig. 1).
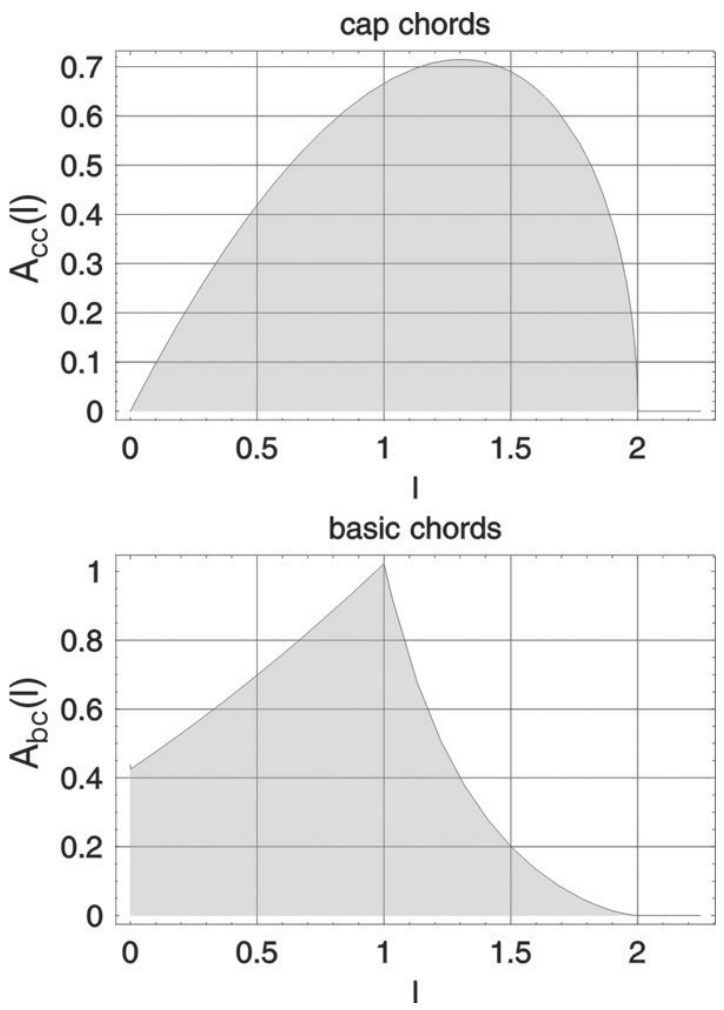

Fig. D2: The functions $A_{c c}(l)$ and $A_{b c}(l)$ for the case $R=1$. In the cc-case $A_{c c}(0)=0$, but in the bccase $A_{b c}(0)>0$. Consequently, $A_{H}(0)>0$, Fig. 2.

Appendix E: The approximation Eq. (9) and its precision Eq. (9) yields $\gamma_{A}{ }^{\prime \prime}(r, R)$,

$$
\begin{aligned}
& \gamma_{A}{ }^{\prime \prime}(r, R)= \\
& \left\{\begin{array}{ccc}
\frac{48}{t \cdot R^{2}}+\frac{3(15 \sqrt{3}+8 \pi-16)}{t} \cdot \frac{r}{R^{3}} & , & 0 \leq r \leq R \\
\frac{3(15 \sqrt{3}+8 \pi)}{t} \cdot \frac{(2 R-r)}{R^{3}} & , & R \leq r \leq 2 R \\
0 & , & 2 R<r
\end{array}\right.
\end{aligned}
$$

Eqs. $(9,15)$ are based on a piecewise linear interpolation of $A_{H}(r, R)$ taking into account the abscissas $r=0, r=R, r=2 \cdot R$ and the limit $r \rightarrow \infty$, followed by normalization via analysis of $\gamma_{A}$ and $A_{A}$. Both, the functions $\gamma_{A}$ "and $A_{A}(l, R)$, Eq. (2), are continuous functions. The strategy of operating with a polynomial approximation of higher degree yields smaller deviations $d(r)=\gamma_{A}(r, R)-\gamma_{H}(r, R)$. However, simultaneously this complicates the integrands in Eq. (8). The effort is not worth it.

Any modification of $\gamma_{H}$ leads to a certain change of the moments of the CLD of the single hemisphere. Analyzing this effect carefully an optimization problem results. A normalization factor, which is indispensable, cannot correct all moments of the CLD simultaneously. Eqs. $(9,15)$ yield an exact 0th moment of the CLD. However, as a consequence of the linearization applied, the higher moments of $A_{A}(l, R)$ differ somewhat from the exact ones. The differences, due to the application of Eq. (9) instead of Eq. (3), are acceptably small,

$$
\begin{aligned}
& \int_{0}^{2 \cdot R} l \cdot A_{H}(l, R) d l=\frac{8}{9} \cdot R, \\
& \int_{0}^{2 \cdot R} l \cdot A_{A}(l, R) d l=\frac{8+45 \sqrt{3}+24 \pi}{3(8+15 \sqrt{3}+8 \pi)} \cdot R .
\end{aligned}
$$

The first moment of $A_{A}(l, R)$ given in the second line of Eq. (16) is an approximation. The deviation is about $2 \%$. The difference $d(r)=\gamma_{A}(r, 1)-\gamma_{H}(r, 1)$ is investigated (Fig. E1).

Eqs. $(3,9)$ have been studied by a Mathematica program. The exact WF is defined in program parts 1 . and 2. Eq. (9) is compiled in parts 3. and 4. Finally, part 5. investigates $d(r)$. The insert of Fig. E1 results.

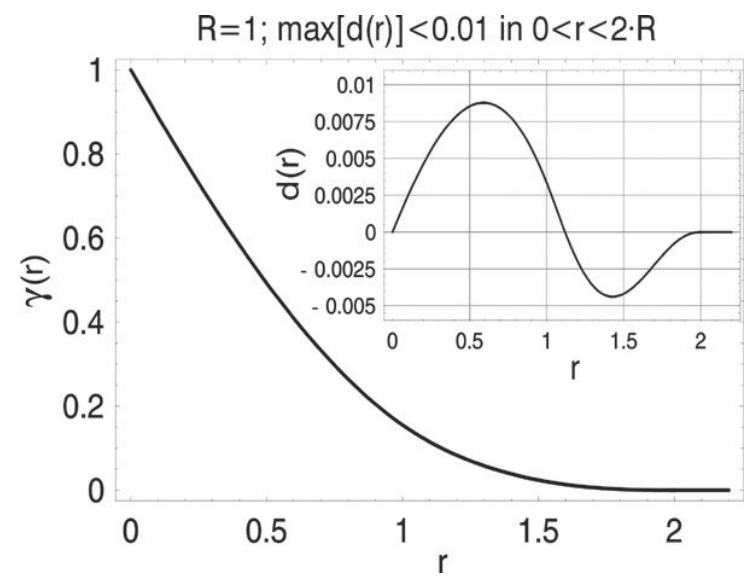

Fig. E1: Verification of the approximation Eq. (9) via analysis of the difference $d(r)$ for $R=1$. The maximum deviation is smaller than $10^{-2}$. 
Clear["Global *"]; Remove["Global *"];

$(* 1$. exact $\mathrm{WF}$ in $0<=\mathrm{r}<=\mathrm{R} *)$

$\mathrm{g} 1\left[\mathrm{r}_{-}, \mathrm{R}_{-}\right]:=\left(3 * \mathrm{r}^{*}\left(\mathrm{r}^{\wedge} 2+2 * \mathrm{R}^{\wedge} 2\right) * \operatorname{Sqrt}\left[-\mathrm{r}^{\wedge} 2+4 * \mathrm{R}^{\wedge} 2\right]+\right.$

$4 * \mathrm{Pi}^{*} \mathrm{r} *\left(\mathrm{r}^{\wedge} 3-9 * \mathrm{r} * \mathrm{R}^{\wedge} 2+8 * \mathrm{R}^{\wedge} 3\right)+24 *(\mathrm{r}-\mathrm{R}) * \mathrm{R} \wedge 2 *(\mathrm{r}+\mathrm{R}) *$

$\operatorname{ArcCsc}[(2 * \mathrm{R}) / \mathrm{r}]) /\left(32 * \mathrm{Pi}^{*} \mathrm{r}^{*} \mathrm{R}^{\wedge} 3\right)$;

(*2. exact $\mathrm{WF}$ in $\mathrm{R}<=\mathrm{r}<=2 \mathrm{R} *$ )

$\mathrm{g} 2\left[\mathrm{r}_{-}, \mathrm{R}_{-}\right]:=\left(3 * \mathrm{r}^{*}\left(\mathrm{r}^{\wedge} 2+2 * \mathrm{R}^{\wedge} 2\right) * \operatorname{Sqrt}\left[-\mathrm{r}^{\wedge} 2+4 * \mathrm{R}^{\wedge} 2\right]+\right.$

$\left.24 * \mathrm{R}^{\wedge} 2 *\left(-\mathrm{r}^{\wedge} 2+\mathrm{R}^{\wedge} 2\right) * \operatorname{ArcSec}[(2 * \mathrm{R}) / \mathrm{r}]\right) /\left(32 * \mathrm{Pi}^{*} \mathrm{r}^{*} \mathrm{R}^{\wedge} 3\right)$;

(*3. approximation in $0<=\mathrm{r}<=\mathrm{R} *$ )

g1 app[r_, R_] $=1+\left(-16 * \mathrm{r}^{\wedge} 3+15 * \operatorname{Sqrt}[3]{ }^{*} \mathrm{r}^{\wedge} 3+\right.$

$\left.8 * \mathrm{Pi}^{*} \mathrm{r}^{\wedge} 3\right) /\left(2 *\left(8+45^{*} \mathrm{Sqrt}[3]+24 * \mathrm{Pi}\right) * \mathrm{R}^{\wedge} 3\right)+$

$\left(24 * \mathrm{r}^{\wedge} 2\right) /\left((8+45 * \operatorname{Sqrt}[3]+24 * \mathrm{Pi}) * \mathrm{R}^{\wedge} 2\right)-$

$(3 *(8+15 * \operatorname{Sqrt}[3]+8 * \mathrm{Pi}) * \mathrm{r}) /$

$((8+45 * \operatorname{Sqrt}[3]+24 * \mathrm{Pi}) * \mathrm{R})$;

(*4. approximation in $\mathrm{R}<=\mathrm{r}<=2 \mathrm{R} *$ )

g2app[r_, R_] $=-(((15 * \operatorname{Sqrt}[3]+8 * \mathrm{Pi}) *$

$\left.\left.(\mathrm{r}-2 * \mathrm{R})^{\wedge} 3\right) /(2 *(8+45 * \operatorname{Sqrt}[3]+24 * \mathrm{Pi}) * \mathrm{R} \wedge 3)\right)$;

(* 5. A plot: approximation of the WF minus exact WF $*)$

$\mathrm{R}=1$;

Plot[Which[0<= r $<$ R, g1app[r, R], $\mathrm{R}<=\mathrm{r}<=2 * \mathrm{R}, \mathrm{g} 2 \mathrm{app}[\mathrm{r}, \mathrm{R}]$, True, 0$]$ Which $[0<=\mathrm{r}<\mathrm{R}, \mathrm{g} 1[\mathrm{r}, \mathrm{R}]$, $\mathrm{R}<=\mathrm{r}<=2 * \mathrm{R}, \mathrm{g} 2[\mathrm{r}, \mathrm{R}]$, True, 0$],\{\mathrm{r}, 0,2.2\}]$;

\section{REFERENCES}

1. Weibel, E.R., 1980. Stereological Methods. Academic Press, London, vol. 2.

2. Stoyan, D., W.S. Kendall and J. Mecke, 1987. Stochastic Geometry and Its Applications. J. Wiley, New York.

3. Serra, J., 1982. Image Analysis and Mathematical Morphology. Academic Press, London.

4. Ohser, J. and F. Mucklich, 2000. Statistical Analysis of Microstructures in Materials Science. J. Wiley and Sons. Chichester, London, New York.

5. Enns, E. and P. Ehlers, 1978. Random paths through a convex region. JAP., 15: 144-152.

6. Hermann, H., 1991. Stochastic Models of Heterogeneous Materials. Trans. Tech. Publications, Zürich.
7. Gille, W., 1983. Stereological characterization of micro-particle systems by use of SAS. Thesis A, Halle (In German).

8. Gille, W., 1995. Diameter Distribution of Spherical Primary Grains in the Boolean Model. Particle \& Particle Syst. Characterization, 12: 123-133.

9. Gille, W., 2000. Chord length distributions and small-angle scattering. Eur. Phys. J.B., 17: 371383.

10. Gille, W., 1999. The Small-Angle Scattering correlation function of a hemisphere. Comp. Mater. Sci., 15: 449-454.

11. Fedorova, I.S and P.W. Schmidt, 1978. A General Analytical Method for Calculating Particle Dimension Distributions from Scattering Data. J. Appl. Cryst., 11: 405-411.

12. Gille, W., 2001. Lecture and programs on CD: Mathematica Programs for Chord Length Distributions of Selected Geometric Figures, PrimMath[2001] Zagreb, Sep. 27-28.

13. Gille, W., 2005. Models for particle sizing of tetrahedral particles. (In Press).

14. Gille, W., 2005. Chord Length Distributions of the Hemisphere. J. Math. Stat., 1: 24-28.

15. Camko, C.G., A. Kulbac and O.I. Maritschew, 1987. Integrals and Products (In Russian). Nauka i Technika, Minsk.

16. Wiener, K., 1999. Private communication.

17. Wolfram Research, 2005. Inc. Mathematica, Champaign, Illinois.

18. Guinier, A. and G. Fournet, 1955. Small-Angle Scattering of X-Rays. J. Wiley, New York.

19. Torquato, S., 2002. Random Heterogeneous Materials. Springer, New York.

20. Dutkiewicz, J., O. Kabisch, W. Gille, O. Simmich, R. Scholz and J. Krol, 2001. Structure changes and precipitation kinetics in melt spun and aged $\mathrm{Al}-\mathrm{Li}-$ $\mathrm{Cu}$ alloy. Z. Metallkd., 92: 1247-1252.

21. Lukac, P., 1997 and 2000. Charies University Prague, Department of Metal Physics. Private communications concerning hemispherical precipitates in $\mathrm{Al}$ alloys and $\mathrm{Mg}$ composites, Zakopane, NATO ASI "Advanced Light Alloys and Composites", 5-15th Sep. 1997 and letter from Prague 2000. 